\title{
Réseau national « métallurgie »
}

\author{
Par François Mudry ${ }^{1}$
}

L'académie des sciences et celle des technologies ont publié un rapport sur la métallurgie en France en 2010. Un comité a été mis en place par le ministère de l'enseignement supérieur et de la recherche afin d'étudier la mise en place des différentes recommandations. Il a fait une série de propositions concrètes début 2014, concernant une structuration de la métallurgie en France avec différents aspects : besoins et organisation de la formation, de la recherche, des relations avec l'industrie et de la visibilité de la discipline. Ce court article les présente rapidement.

\section{Le rapport des académies sur la métallurgie}

L'académie des sciences et l'académie des technologies ont rédigé un rapport dit « RST » sur la métallurgie en France, intitulé « La métallurgie Science et Ingénierie », approuvé en juillet 2010. Ce travail attirait l'attention des pouvoirs publics sur le danger d'une perte de compétence irrémédiable de la France dans cette discipline en raison d'une part, du non-remplacement des spécialistes académiques du domaine au fur et à mesure de leur départ en retraite et, d'autre part, de l'éloignement des centres de décision des différentes industries productrices de métaux, du fait de la globalisation de l'économie, même si, pour l'instant, les centres de recherche restent sur le territoire national, il y a un risque réel de les voir fermer. Pourtant, dans le même temps, des industriels de secteurs utilisateurs tels que l'aéronautique, l'automobile ou la production d'énergie, se plaignent du manque de métallurgistes qualifiés pour affronter les défis auxquels ils sont confrontés. La désaffection des partenaires académiques pour cette discipline provient de son image " ringarde » auprès des étudiants et du grand public, largement due à la médiatisation importante de quelques fermetures d'usine spectaculaires. À leur départ, les professeurs, de métallurgie sont remplacés par des

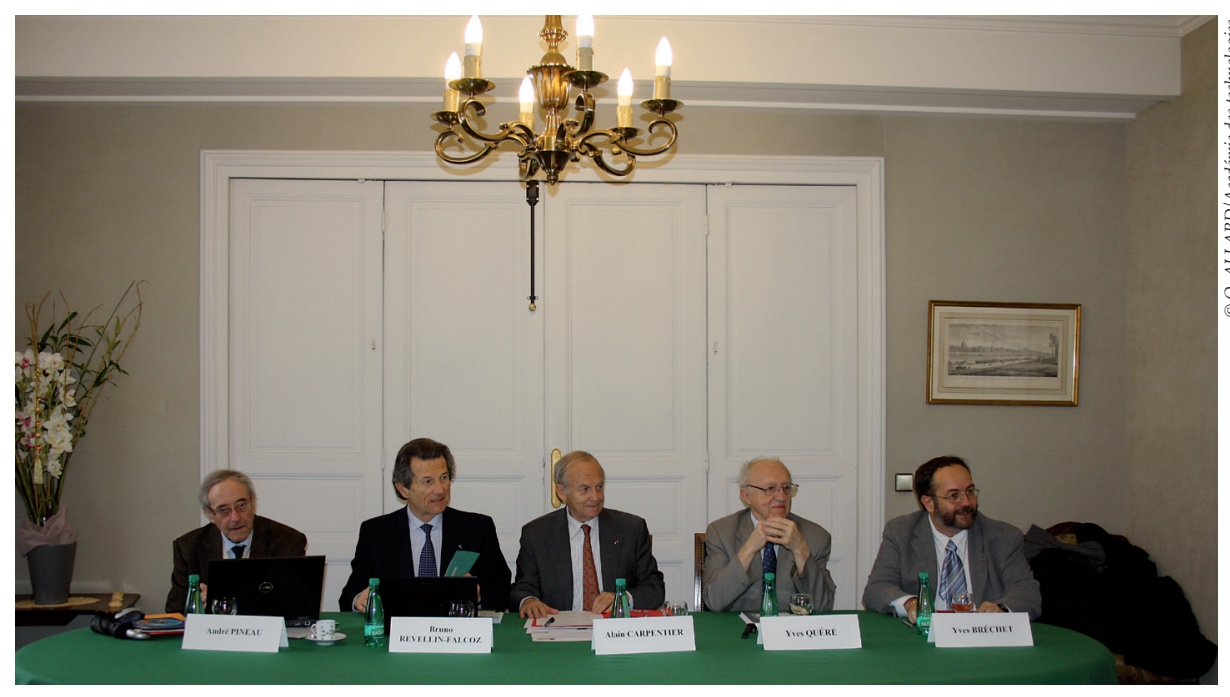

Intervenants de la conférence de presse du 20 janvier 2011 à l'Académie des sciences «L'avenir de la métallurgie française en danger. De gauche à droite : André Pineau, Académie des technologies, Bruno Revellin-Falcoz, Académie des technologies (Président 2011-2012), Alain Carpentier, Académie des sciences (Président 2011-2012), Yves Quéré, Académie des sciences et Yves Bréchet, Académie des sciences.

spécialistes d'autres disciplines considérées comme plus excitantes sur le plan scientifique et plus valorisantes d'un point de vue communication. C'est pourquoi, une partie importante du rapport des académies présentait toute la richesse des développements scientifiques récents, les questions ouvertes, ainsi que la variété insoupçonnée des domaines applicatifs concernés, démontrant ainsi que l'image " ringarde » de la discipline ne reflète absolument pas la réalité. La suite du rapport insistait sur la variété des applications dans différentes branches industrielles en insistant sur leurs enjeux considérables, notamment dans la perspective d'une transition économique vers un développement durable. Le rapport fait en outre quelques recommandations précises. Il plaide pour la remise en place d'une politique industrielle dont la métallurgie ne serait qu'un volet, rejoignant en cela une évolution politique récente, tant au niveau national qu'au niveau de l'Europe. Il propose de réintégrer des cours de métallurgie dans les écoles d'ingénieur, IUT et BTS, comme matière introductive à la science des matériaux source 


\section{Événement}

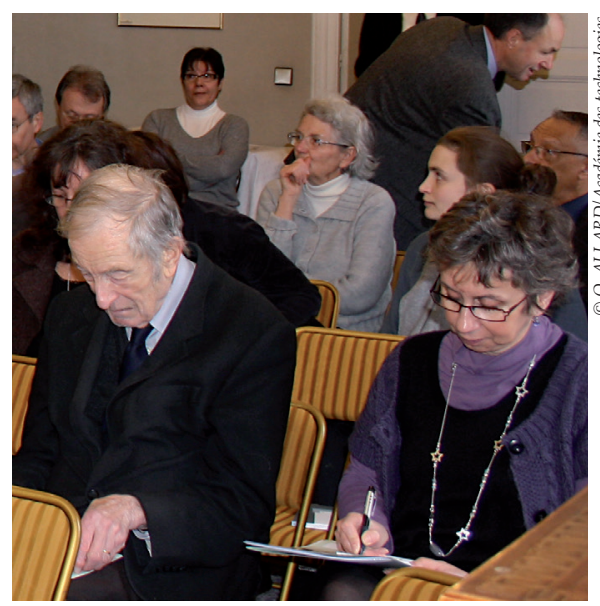

Jacques Friedel qui nous a malheureusement quittés récemment, lors de la conférence de presse du 20 janvier 2011 à l'Académie des sciences «L'avenir de la métallurgie française en danger $»$.

de nombreuses applications technologiques ; de redonner une place visible à la discipline dans les différents organismes de recherche où elle se trouve éclatée entre différentes directions. Il recommande enfin de mettre en place une « mission métallurgie » chargée de coordonner au niveau national la recherche académique, la formation et la recherche industrielle. Il suggère d'installer 2 à 4 pôles d'enseignement / recherche au niveau national pour affermir les collaborations recherches académique/formation/centres de recherche industriels.

\section{Les travaux du comité d'orientation nationale de la métallurgie}

Quatre ans après, où en sommesnous? Les conclusions du rapport ont été présentées à de multiples instances industrielles, académiques et gouvernementales en 2010 et 2011. Vers la fin de l'année 2011, le ministère de l'enseignement supérieur et de la recherche a mis en place une mission confiée à Jean-Jacques Maillard, conformément à l'une des recommandations du rapport. Le ministère de l'industrie n'a pas souhaité s'associer officiellement mais a marqué son intérêt pour ses conclusions et a délégué un représentant pour suivre les travaux de la commission et être régulièrement informé. La mission, intitulée : "Comité d'orientation national sur la métallurgie » a été confirmée le 18 avril 2012 après le changement de gouvernement. Elle était composée de représentants des universités, du CNRS, du CEA, de l'ONERA et de plusieurs industries productrices ou utilisatrices de métaux. Elle a travaillé par groupes : l'un sur la structuration en 3 pôles au plan national ; un sur la « visibilité » et le déficit d'image de la métallurgie, un sur les besoins anticipés en recrutement, un dédié à la formation, un concernant la façon de coordonner les industriels et les tutelles des laboratoires et un, enfin, concernant la recherche.

Le comité a remis ses conclusions tout début 2014. Celles-ci ont été présentées à madame la ministre de l'enseignement supérieur et de la recherche qui s'est montrée intéressée et a donné son accord pour la mise en place des principales conclusions. Elles ont été présentées au cours d'une réunion tenue à Paris en octobre 2013 à laquelle assistaient des représentants de presque tous les centres de recherche académiques en France.

\section{Les principales conclusions des travaux}

\section{Les pôles structurants et les centres d'excellence}

Afin de structurer la communauté scientifique de la métallurgie de façon lisible vis-à-vis des différents acteurs, tant du point de vue académique qu'industriel, il est proposé de confier des responsabilités d'animation à trois pôles structurants. Chacun de ces pôles concentrera ses efforts sur l'une des thématiques cœur de la métallurgie : - métallurgie de l'élaboration et de la transformation : métallurgie extractive, élaboration, solidification, traitements thermiques, traitements de surface,
- métallurgie physique (structure des alliages) : transformations de phases, physique de la plasticité, utilisation des grands instruments,

- métallurgie mécanique (propriétés mécaniques des alliages) : mécanique de la rupture et de l'endommagement, formulation de nouveaux alliages, comportement sous irradiation, fatigue thermomécanique

Cette structuration nationale autour des pôles Lorrain, Rhône Alpin et Francilien, s'appuie naturellement sur un réseau de centres d'excellence, importants au plan national, aux compétences scientifiques reconnues dans des domaines particuliers de la métallurgie, en lien étroit avec le tissu industriel local.

Les compétences évolueront ainsi dans le cadre d'une coordination reposant nécessairement sur des relations confiantes sur la durée. Les pôles structurants acceptent de concentrer leurs efforts sur chacune des thématiques précisées ci-dessus. Les centres d'excellence, répartis sur tout le territoire, contribueront aux projets de rechercher dans leur domaine d'expertise et permettront de maintenir un enseignement de haut niveau de la métallurgie en s'appuyant sur les synergies dégagées dans le réseau.

C'est en ce sens que le projet de création d'un « réseau national de la métallurgie », comme une commission de la Société Française de Métallurgie et de Matériaux (SF2M), a été validé par le conseil d'administration de la SF2M. Ce réseau sera chargé, en s'appuyant sur des moyens humains limités, de l'animation et de la mise en place des recommandations du Comité.

\section{Concernant la recherche}

Sur le plan de la recherche, le comité a défini certaines compétences indispensables à une recherche nationale de qualité. Elles sont indiquées ci-dessous. C'est autour d'elles qu'il faut assurer une coordination nationale en utilisant les procédures de soutiens à la recherche : projets européens, 
projets nationaux (Equipex, ANR, ADEME), régionaux :

- métallurgie extractive et recyclage,

- métallurgie de la formulation, métallurgie de l'élaboration,

- métallurgie de la transformation et de la mise en forme,

- métallurgie physique,

- métallurgie mécanique et propriétés d'usage,

- tenue aux agressions chimiques de l'environnement,

- assemblage,

- traitement de surface,

- métallurgie spéciale (autres propriétés physiques, irradiation...).

\section{Les besoins en formation}

Une étude prospective sur l'évolution des emplois et des métiers de la métallurgie a été réalisée en 2012 afin d'anticiper les besoins en compétences à l'horizon 2015-2020. Il en ressort que : - l'ensemble des secteurs de la métallurgie qui recouvrent la fabrication et l'utilisation des métaux représente 1,5 million d'emplois,

- les prévisions de recrutement resteront à un niveau élevé : de 115000 à 128000 par an d'ici 2020 suivant les scénarios économiques,

- dans le seul secteur " métallurgie et fabrication de produits métalliques ", 31500 recrutements sont prévus en 2020,

- une hausse des besoins est prévue pour les catégories ingénieurs et cadres techniques (23 660 en 2020 soit $+10 \%$ par rapport à 2010) et techniciens et agents de maîtrise (20 360 soit $+3,8 \%$ par rapport à 2010 ) alors que la baisse des besoins de recrutement concerne essentiellement les ouvriers non qualifiés (21 720 en 2020 soit $-40 \%$ par rapport à 2010 ).

Il est recommandé de faire une étude plus précise pour évaluer les besoins réels de formation de façon plus fine au niveau des compétences et type d'enseignement. La mise en place d'une telle étude, avec le soutien des pouvoirs publics, serait un des objectifs du réseau.

\section{François Mudry, Président de I'IRT-M2P}

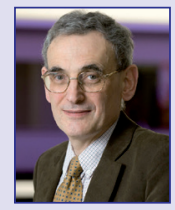

François Mudry, ingénieur des mines, docteur en métallurgie, ancien directeur des recherches de l'École des Mines de Paris, ancien directeur scientifique d'Arcelor Mittal, est présentement président de l'IRT-M2P.

L'IRT M2P est un Institut de Recherche Technologique issu du Programme d'Investissements d'Avenir soutenu par l'État.

L'institut vise à devenir un centre commun de recherche technologique associant autour de plusieurs plateaux techniques, entreprises, établissements et organismes de recherche, principalement dans l'étape de recherche industrielle, transition entre la recherche fondamentale et le développement expérimental.

Les travaux de l'IRT M2P couvrent l'ensemble de la chaîne de production des matériaux principalement métalliques, depuis les matières premières (avec en particulier le recyclage) jusqu'aux produits, associations de matériaux (et à leurs fonctions), à travers leurs procédés d'élaboration dans un contexte de développement durable (économies d'énergie et de matières premières, durabilité des produits).

Source : www.irt-m2p.eu

\section{L'organisation de la formation}

Sur le plan de la formation, le comité propose plusieurs pistes de réflexion tant sur les méthodes de formation (privilégier la formation par projets, par exemple) que sur les différents publics visés (spécialistes et experts, ingénieurs généralistes, techniciens supérieurs, formation continue, en alternance ou par apprentissage). Il insiste sur la nécessité de partager et de mutualiser les outils de formation. En particulier, il recommande que le réseau de la métallurgie organise des cours de « formation des formateurs " pour les professeurs d'IUT et BTS et qu'il pose sa candidature pour construire une formation numérique en métallurgie. Il propose également : des séjours longs à l'étranger pour les étudiants, hors d'un système contraignant de double diplôme, permettant d'acquérir des compétences complémentaires auprès des meilleurs spécialistes d'un domaine ; la mise en place d'écoles d'été européennes sur des thèmes spécifiques ; la création d'un forum annuel sur les matériaux permettant de sensibiliser la communauté sur les changements dans le domaine et leur impact sur la formation.

\section{L'implication des industriels}

Pour l'implication des industriels, il est prévu que le réseau national de la métallurgie soit financé par une augmentation modeste des cotisations à la SF2M pour les laboratoires académiques membres du réseau et les industriels intéressés par l'organisation au niveau national de la métallurgie. Il est également prévu de mettre en place une structure nationale de coordination entre les industriels et les tutelles des laboratoires académiques de façon à porter un diagnostic commun sur l'évolution des compétences en France afin de définir, si nécessaire, des actions correctrices coordonnées en utilisant les moyens disponibles pour chacun des partenaires : post-docs, chaires industrielles, accords-cadres, contrats de recherche spécifiques, etc.

\section{La communication : visibilité de la métallurgie}

Enfin sur le plan de la visibilité de la métallurgie, plusieurs actions sont recommandées à prendre en charge par les académies et le réseau national. Elles sont orientées vers différentes cibles : les enfants du primaire, les professeurs des Écoles, les élèves et les professeurs du secondaire, le grand public et le Parlement.

\section{En guise de conclusion}

$C^{\prime}$ est donc tout un plan de revitalisation de la métallurgie en France qui est proposé avec un déploiement de moyens relativement modestes. Ceci devrait permettre d'enrayer la décroissance constatée ces dernières années et de repartir de l'avant comme le dynamisme de la discipline et l'importance des enjeux industriels nous y incitent. 